\title{
Le cycle biologique de Maritrema pyrenaica
}

\author{
Deblock et Combes, 1965 \\ Parasite de micromammifères insectivores pyrénéens
}

\author{
par J. JOURDANE \\ Département de Biologie animale (Dir. Pr C. Combes), \\ Université de Perpignan, av. de Villeneuve, F 66025 Perpignan Cedex.
}

\section{Résumé.}

L'auteur rapporte en détail les modalités du cycle évolutif de Maritrema pyrenaica. Au stade sporocyste, ce Digène parasite le Mollusque Prosobranche Bythinella reyniesii. La xiphidio-cercaire, du type monostome, anentérique, est libérée dans l'eau et pénètre activement chez le Crustacé Gammarus pulex où elle s'enkyste.

Au plan épidémiologique, les foyers à $M$. pyrenaica sont formés de deux aires : l'endémiotope (aire de productivité parasitaire) correspondant au biotope à Bythinella, et l'aire de dispersion, représentée par la longueur de la vallée où s'observe une positivité du Vertébré.

\section{Summary.}

The life cycle of Maritrema pyrenaica Deblock et Combes, 1965, a parasite of Insectivorous Micromammals in the Pyrenees.

The life cycle of Maritrema pyrenaica is described. M. pyrenaica develops to the sporocyst stage in the snail Bythinella reyniesii. The xiphidio-cercaria, monostomous, anenterous in type, is liberated in water and actively penetrates a Crustacea Gammarus pulex where it encysts.

From the point of view of epidemiology, the focuses of $M$. pyrenaica are divided into two different areas: the area where the parasites multiply (that we call "l'endemiotope ») coinciding with the Bythinella biotope and the area limited to the length of the river where the Vertebrate is parasitized.

Accepté le 15 mars 1979. 
L'espèce Maritrema pyrenaica a été décrite à partir de matériel récolté chez un Desman des Pyrénées (Galemys pyrenaica Geoffroy) capturé dans une localité de l'est des Pyrénées (Deblock et Combes, 1965). Retrouvé dans cette même station (Combes, Jourdane et Théron, 1976) chez un autre Micromammifère Insectivore (Neomys fodiens), ce Digène a été recensé depuis par nous-mêmes chez ce dernier hôte dans plusieurs autres biotopes des Pyrénées orientales et centrales. Une étude parasitologique des Invertébrés les plus couramment rencontrés dans ces mêmes stations nous a permis d'élucider parallèlement le cycle évolutif de ce Microphallidé. Nous livrons ci-après les modalités de son évolution.

\section{Schéma du cycle}

Nos recherches nous permettent de résumer le cycle biologique de $M$. pyrenaica comme suit :

- l'œuf, éliminé avec les fèces dans l'eau, est ingéré par le Mollusque Hydrobiidé Bythinella reyniesii;

- le miracidium, libéré dans le tube digestif du Mollusque, évolue chez celui-ci en sporocyste I qui donne naissance à son tour à des sporocystes II parasitant les glandes digestive et génitale ;

- la cercaire, formée dans le sporocyste II, quitte le Mollusque et nage à la recherche du deuxième hôte, Gammarus pulex, chez lequel elle pénètre activement et s'enkyste ;

- le Mammifère Insectivore s’infeste après ingestion des Gammares porteurs de métacercaires.

\section{Stades larvaires}

\section{Sporocyste.}

Nous n'avons pu mettre en évidence les sporocystes primaires. Les sporocystes secondaires (fig. $1 \mathrm{~A}$ ) parasitent principalement les glandes digestive et génitale du Mollusque. Dans quelques cas, nous en avons aussi localisé dans la cavité branchiale. Ils se présentent sous la forme de sacs incolores, ellipsoïdes, adhérant de façon très lâche aux tissus de l'hôte. Ils renferment à la fois des cercaires différenciées, très mobiles, et des bourgeons cercariens à tous les stades de l'embryogenèse. Le nombre de formes larvaires par sporocyste varie entre 10 et 30 . Les sporocystes mesurent 290-400 $\mu \mathrm{m} \times 80-100 \mu \mathrm{m}$ (moyenne : $330 \times 90 \mu \mathrm{m}$ ).

\section{Cercaire.}

Morphologie générale ( $f$ ig. $1 \mathrm{~B}$ et $3 \mathrm{~A}$ ). 

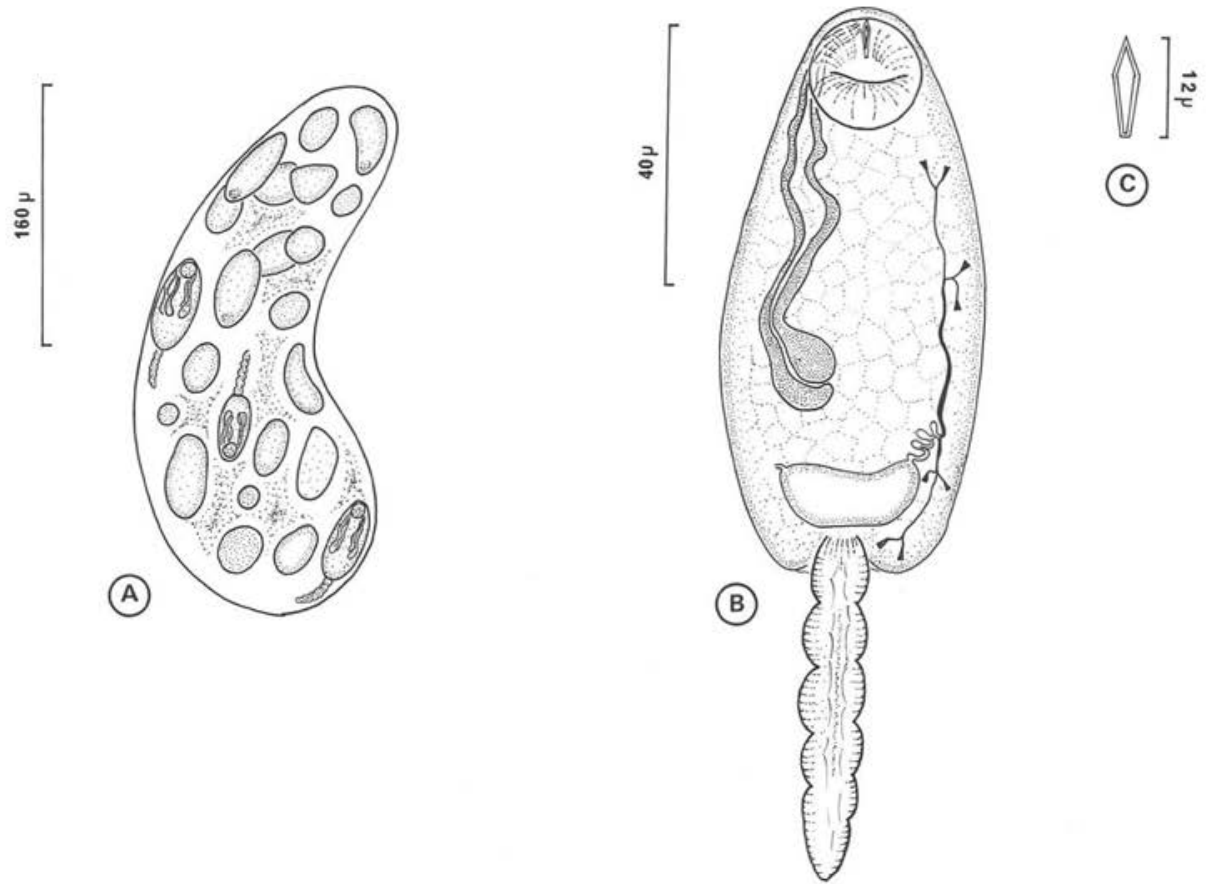

Fig. 1. Maritrema pyranaica Deblock et Combes, 1965. A : Sporocyste fils montrant des cercaires en voie de différenciation. B : Cercaire, morphologie générale (vue ventrale). C: Stylet de la cercaire

La cercaire se range dans le groupe des cercaires xiphidiocerques, monostomes, anentériques, leptocerques.

Le corps a la forme d'un ovale assez régulier. Son tégument est glabre.

La queue, de longueur très variable selon son état de contraction, s'insère en position ventrale à l'extrémité postérieure du corps. La ventouse orale, subterminale et ventrale, de forme circulaire, est armée d'un stylet (fig. 1C) implanté sur sa lèvre antérieure. Celui-ci a la forme d'un fer de lance. Les dimensions rapportées ci-dessous correspondent à celles de 20 larves vivantes mesurées entre lame et lamelle sans aplatissement :

- longueur du corps : 70-110 $\mu \mathrm{m}(80 \mu \mathrm{m})$;

- largeur du corps : 35-60 $\mu \mathrm{m}(50 \mu \mathrm{m})$;

- longueur de la queue contractée : $30-50 \mu \mathrm{m}(40 \mu \mathrm{m})$;

- largeur de la queue contractée : 10-20 $\mu \mathrm{m}(15 \mu \mathrm{m})$;

— diamètre de la ventouse orale : 15-25 $\mu \mathrm{m}(20 \mu \mathrm{m})$;

- longueur du stylet : 11-12 $\mu \mathrm{m}(12 \mu \mathrm{m})$. 

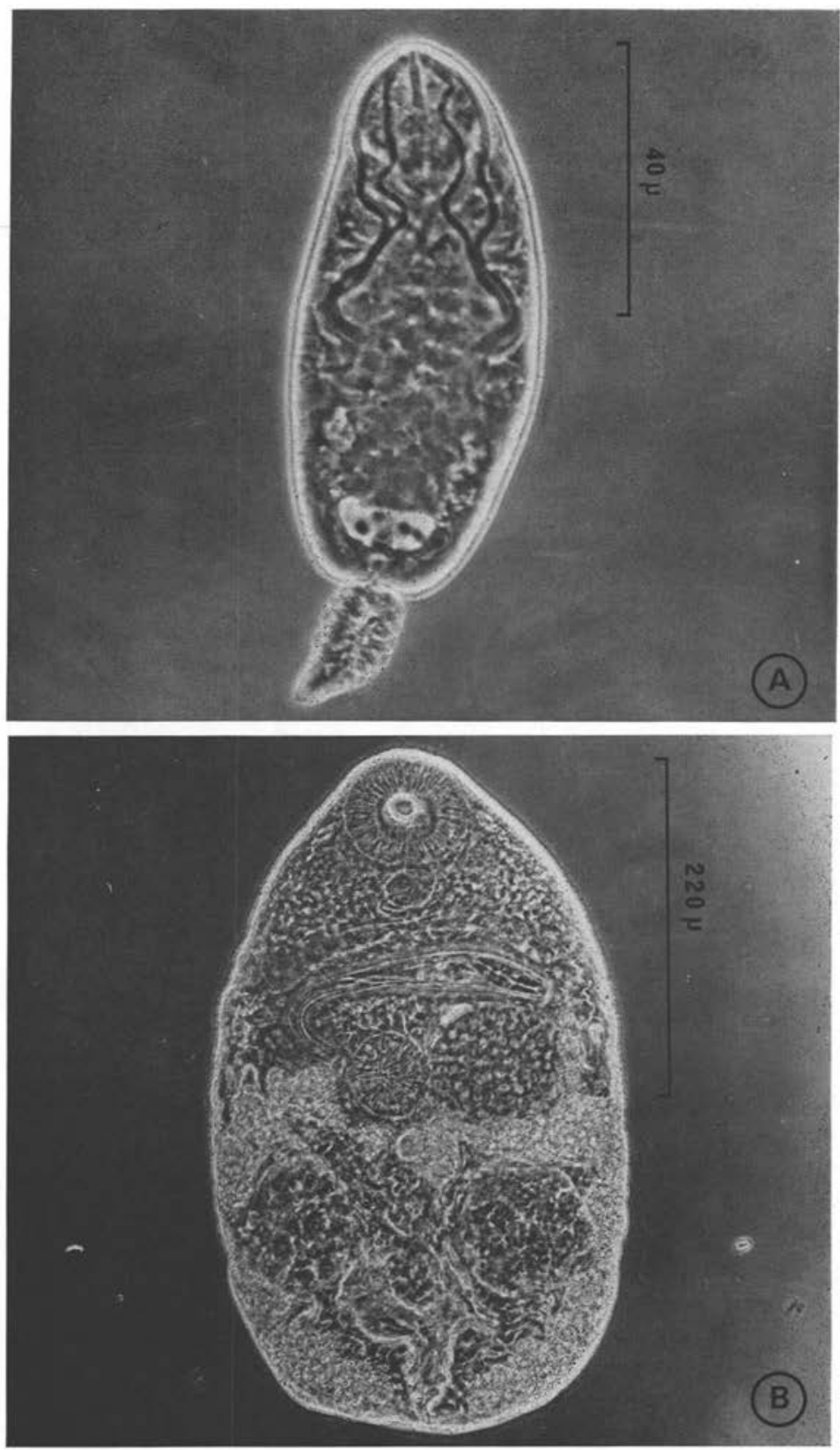

Fig. 2. Maritrema pyrenaica Deblock et Combes, 1965. A : Cercaire photographiée en contraste de phase. B : Métacercaire dékystée depuis quatre heures, photographiée en contraste de phase (vue dorsale) 
Organisation interne.

La cercaire est dépourvue de tube digestif.

L'appareil glandulaire de pénétration comprend deux paires de glandes unicellulaires disposées symétriquement de part et d'autre de l'axe sagittal de la cercaire. Les corps cellulaires sont situés au niveau du deuxième tiers de la longueur du corps cercarien. Leurs canaux remontent en direction de la ventouse orale selon un trajet sinueux et débouchent sur sa lèvre antérieure, de part et d'autre de la pointe du stylet.

Le système excréteur comprend une vessie bilobée et huit paires de cellules flammes groupées deux par deux selon la formule :

$$
2[(2+2)+(2+2)]=16 .
$$

\section{Comportement.}

Les cercaires de $M$. pyrenaica sont émises selon un rythme circadien pendant la phase obscure du nycthémère. Dans les conditions de maintenance au laboratoire, la cercaire a une durée de vie courte, de l'ordre de 2 heures. Elle n'est de plus vraiment active que pendant la première heure d'émergence. La pénétration de la cercaire dans le corps du deuxième hôte se réalise préférentiellement au niveau des articulations des appendices.

\section{Métacercaire.}

Les métacercaires se distribuent en amas dans la cavité générale du Crustacé entre les diverticules intestinaux. Chez les Gammares infestés dans les conditions naturelles, ces métacercaires sont associées à celles de Microphallus gracilis, espèce sympatrique également parasite de la Musaraigne aquatique.

Le kyste de la métacercaire a la forme d'un ovale régulier de 230-300 $\mu \mathrm{m}$ $\times 170-230 \mu \mathrm{m}(270 \times 200 \mu \mathrm{m})(f i g .3 \mathrm{~A})$. Sa paroi est relativement épaisse $(12 \mu \mathrm{m})$. Elle est constituée de deux enveloppes d'inégale épaisseur et de nature différente : l'enveloppe externe, de nature mucopolysaccharidique, mesure $3,5 \mu \mathrm{m}$, l'enveloppe interne, hyaline, a une épaisseur de $8,5 \mu \mathrm{m}$.

La larve est fortement repliée à l'intérieur du kyste. Les organes digestifs et génitaux y sont parfaitement identifiables.

Nous avons représenté sur la figure 3 B la larve fraîchement dékystée : son organisation anatomique est absolument conforme à celle de l'adulte. A l'état frais, sans aplatissement, nous avons relevé les dimensions suivantes chez les métacercaires dékystées (fig. 2 B) :

- longueur du corps : 350-550 $\mu \mathrm{m}(430 \mu \mathrm{m})$;

- largeur du corps : 230-300 $\mu \mathrm{m}(280 \mu \mathrm{m})$;

- ventouse orale : $80-100 \mu \mathrm{m} \times 70-80 \mu \mathrm{m}(90 \times 70 \mu \mathrm{m})$;

- diamètre de la ventouse ventrale : 55-65 $\mu \mathrm{m}(60 \mu \mathrm{m})$; 
- pharynx $(28 \times 26 \mu \mathrm{m})$;

- longueur des caecums : 90-140 $\mu \mathrm{m}(120 \mu \mathrm{m})$;

— testicules : 80-120 $\mu \mathrm{m} \times 60-95 \mu \mathrm{m}(110 \times 75 \mu \mathrm{m})$;

- ovaire : $90-140 \mu \mathrm{m} \times 55-85 \mu \mathrm{m}(100 \times 70 \mu \mathrm{m})$;

- poche du cirre : $165-210 \mu \mathrm{m} \times 20-35 \mu \mathrm{m}(185 \times 30 \mu \mathrm{m})$.
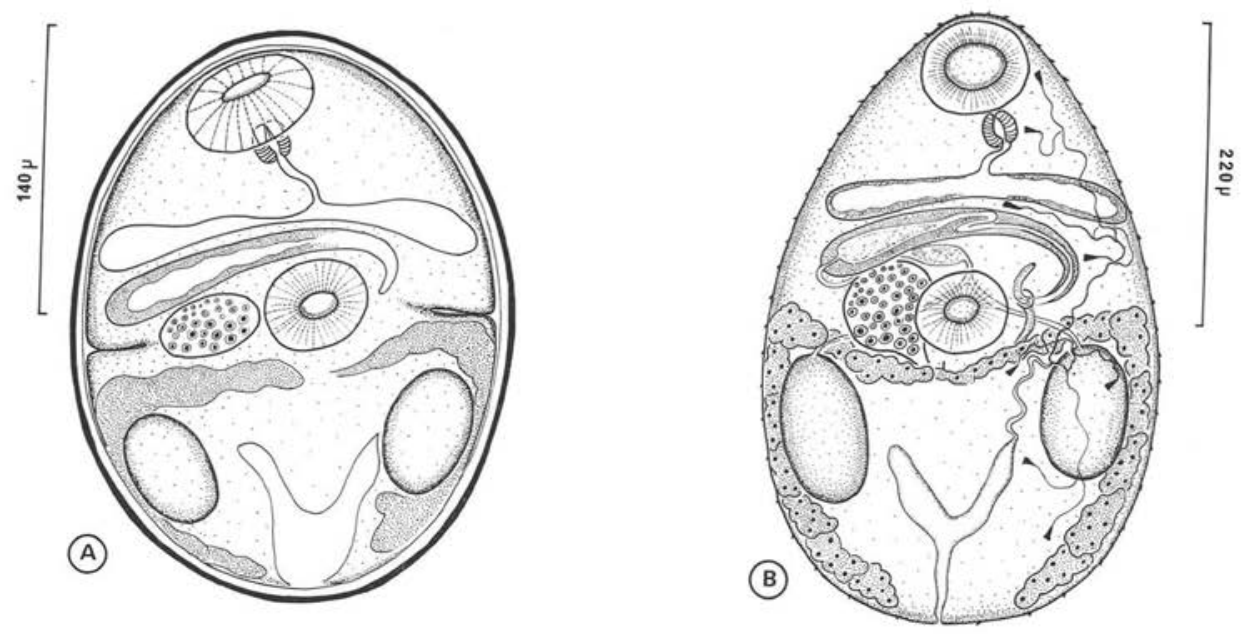

Fig. 3. Maritrema pyrenaica Deblock et Combes, 1965. A : Métacercaire enkystée chez Gammarus pulex. B : Métacercaire dékystée in vitro depuis quatre heures; morphologie générale (vue ventrale)

\section{Recherches expérimentales}

Nous avons testé l'infectivité de la cercaire à l'égard de plusieurs Invertébrés systématiquement rencontrés dans les biotopes où sévit la parasitose :

- Ephéméroptères (Ecdyonurus sp., Ephemerella sp.);

- Trichoptères (Potamophylax cingulatus, Limnephilus sp.);

- Plécoptères (Perla marginata, Isoperla pyrenaica) ;

- Crustacés (Cyclops sp., Gammarus pulex).

Seules les expériences réalisées avec l'Amphipode Gammarus pulex ont été positives. A la température moyenne de l'eau enregistrée dans les foyers du parasite $\left(10^{\circ} \mathrm{C}\right)$, le développement de la métacercaire jusqu'au stade infestant se fait en 30 jours. 
Nous n'avons pu tenter, pour des raisons matérielles, la transmission de la parasitose à l'hôte Vertébré. Néanmoins, la maturation de la métacercaire jusqu'au stade ovigère peut être aisément déclenchée in vitro en 72 h (dans le liquide de Ringer, à $38^{\circ} \mathrm{C}$ ). Les Digènes ainsi obtenus s'identifient sans aucune réserve aux individus de $M$. pyrenaica récoltés dans les conditions naturelles chez le Desman ou la Musaraigne aquatique.

\section{Modalités du cycle dans la nature}

Le cycle du Digène Maritrema pyrenaica se déroule dans la nature chez les hôtes suivants :

— premier hôte intermédiaire : Bythinella reyniesii ;

- deuxième hôte intermédiaire: Gammarus pulex ;

- hôtes définitifs: Galemys pyrenaica, Neomys fodiens.

Le Mollusque B. reyniesii (Prosobranche Hydrobiidae) est une espèce endémique des Pyrénées, recensée exclusivement dans la zone des sources froides de montagne (zone du crenon). Les stations où il a été trouvé parasité par $M$. pyrenaica sont toutes constituées par des résurgences sur terrain calcaire. Son taux de contamination oscille entre 1 et $5 \%$.

Le deuxième hôte, $G$. pulex, est aussi très localisé dans la chaîne. Il est totalement absent de la zone axiale cristalline. Seuls les cours d'eau très calcaires abritent ce Crustacé. La fréquence de la parasitose chez cet hôte varie beaucoup selon les bassins versants : de 40 à $100 \%$.

Deux Vertébrés hébergent $M$. pyrenaica dans les Pyrénées: $G$. pyrenaica et $N$. fodiens. La densité de parasites par hôte dans les zones d'endémie peut être très importante (jusqu'à 100 parasites). Le nombre de vallées pyrénéennes où sévit la parasitose est très limité. Ce caractère trouve son explication dans le fait que l'évolution du parasite à l'état larvaire se réalise chez deux hôtes intermédiaires aux exigences écologiques très strictes: $B$. reyniesii et $G$. pulex.

Il est intéressant de noter que $M$. pyrenaica est toujours associé chez $N$. fodiens à un autre Microphallidé, Microphallus gracilis, qui évolue chez les mêmes hôtes intermédiaires. Néanmoins, l'effectif des populations de ces deux Digènes chez la Musaraigne dans un même foyer est très différent. Le rapport des populations, voisin de $1 / 2$, est dans certaines stations en faveur de $M$. pyrenaica, dans d'autres en faveur de $M$. gracilis. Chez l'hôte vecteur, le rapport des populations métacercariennes des deux espèces est en concordance parfaite avec celui des populations d'adultes dans la même station. Ce caractère épidémiologique atteste de l'existence d'un processus d'exclusion partielle soit de l'un, soit de l'autre des Digènes, dont le mécanisme n'apparaît pas à l'évidence. 


\section{Conclusion}

Un seul cycle de Microphallidé parasite de Mammifère était entièrement connu jusqu’ici, celui de Microphallus gracilis. Maritrema pyrenaica représente donc le deuxième Microphallidé de Mammifère dont l'évolution est élucidée dans sa totalité.

Son cycle de développement, superposable à celui de $M$. gracilis, s'inscrit sans aucune réserve dans le cadre des processus de transmission connus chez les Microphallidae :

- le premier hôte intermédiaire est un Prosobranche de la famille des Hydrobiidae ;

- le deuxième hôte intermédiaire est un Crustacé.

Au plan de l'écologie de la transmission, $M$. pyrenaica ne montre pas de divergences marquées avec les données fournies par les six cycles de Digènes de $N$. fodiens évoluant dans les Pyrénées chez le même premier hôte intermédiaire, B. reyniesii. On peut reconnaître au sein du foyer épidémiologique les deux aires fondamentales décrites pour les six espèces (Jourdane, 1976) :

- l'endémiotope, ou aire de production parasitaire, représentée par les points où les aires de distribution des trois hôtes du cycle se superposent;

- l'aire de dispersion parasitaire, qui recouvre la longueur des bassins versants où s'observe une positivité du Vertébré.

Dans notre région, la rareté des foyers à $M$. pyrenaica et à $M$. gracilis contraste de façon marquée avec la fréquence des autres distomatoses de $N$. fodiens évoluant chez $B$. reyniesii. $\mathrm{Si}$, pour ces Digènes, la plupart des localités à $B$. reyniesii constituent en effet des endémiotopes du fait de la large distribution du ou des hôtes vecteurs, pour $M$. pyrenaica et $M$. gracilis, l'extrême localisation du deuxième hôte G. pulex limite de façon considérable le nombre de stations pouvant fonctionner comme endémiotopes.

\section{Bihliographie}

Combes C., Jourdane J., Théron A. (1976): Données nouvelles sur la faune des Digènes parasites de Neomys fodiens dans les Pyrénées. Vie Milieu, 26, 133-142.

Deblock S., Combes C. (1965) : Contribution à l'étude des Microphallidae, Tranassos, 1920 (Trematada). X. Maritrema pyrenaica n. sp., parasite du Desman (Mammifère Insectivore). Essai de clé diagnostique des espèces du genre. Bull. Soc. Zool. Fr., 90, 101-117.

Jourdane J. (1976) : Ecologie du développement et de la transmission des Plathelminthes parasites de Soricidae pyrénéens. Mém. Mus. nat. Hist. nat., série A, 103, 1-171. 